\title{
FACILE SYNTHESIS OF CdS NANOCRYSTALS USING THIOGLYCOLIC ACID AS A SULFUR SOURCE AND STABILIZER IN AQUEOUS SOLUTION
}

\author{
Yilin Wang ${ }^{*}$, Jianping Lu, Zhangfa Tong, Bin Li and Liya Zhou \\ College of Chemistry and Chemical Engineering, Guangxi University, Nanning 530004, PR \\ China
}

(Received October 1, 2010; revised July 4, 2011)

\begin{abstract}
A novel method has been developed for the synthesis of CdS nanocrystals (NCs) using thioglycolic acid (TGA) as a sulfur source and stabilizer with the presence of hydrogen peroxide in an aqueous medium. The products were characterized by X-ray powder diffraction (XRD), transmission electron microscopy (TEM) and Fourier transform infrared (FTIR) spectroscopy. The results indicate that the product was of zincblend crystal structure in a sphere-like shape. The room-temperature luminescence spectra revealed that the emission peak of CdS NCs prepared in relatively short refluxing times (10-120 min) could be tuned from $518 \mathrm{~nm}$ to $610 \mathrm{~nm}$, and the photoluminescence quantum yield of the as-prepared CdS NCs could reach as high as $12.6 \%$. In addition, the mechanism of the $\mathrm{CdS}$ nanocrystals formation was preliminarily discussed.
\end{abstract}

KEY WORDS: Chemical synthesis, CdS, Nanostructures, Optical properties, Photoluminescence spectroscopy

\section{INTRODUCTION}

CdS nanocrystals (NCs) have gained significant attention in the past decade because of their unique optical properties, and extensive researches have been focused on the synthesis of CdS NCs [1-4]. Based on these researches, the synthesis of CdS NCs can be summarized in three methods according to their chemical routes, the first is the nonaqueous trioctyl phosphine/trioctyl phosphine oxide (TOP/TOPO) technique, the second is the reverse micelle method, and the third is using different thiols as stabilizing agents in aqueous solution. The most popular route for the synthesis of high quality $\mathrm{CdS} \mathrm{NCs}$ is the nonaqueous technique, which was originated by Murray [1] and developed by Peng [2]. However, a further process is needed for the CdS NCs used in a biological system due to as-prepared CdS NCs often dispersing in nonpolar organic solvents. The water-soluble CdS NCs were prepared in an aqueous solution using thiols as stabilizing agents, which was first reported by Weller's group [4]. Up to now, many methods for preparing $\mathrm{CdS}$ NCs in an aqueous solution have been developed [5-8]. Of them, sodium sulfide [9], thiourea [10] and thioacetamide [11] were often used as sulfur sources. In this report, a novel method has been developed for the synthesis of CdS NCs using thioglycolic acid (TGA) as a sulfur source and stabilizer with the presence of hydrogen peroxide in an aqueous environment.

\section{EXPERIMENTAL}

\section{Materials}

Cadmium chloride hemidihydrate (99\%), thioglycolic acid (TGA, 90\%), hydrogen peroxide (30\%) and sodium hydroxide (96\%) were obtained from Sinopharm Chemical Reagent Co. Ltd. (China). All chemicals were used as received; doubly deionized water was used throughout the experiments.

\footnotetext{
*Corresponding author. E-mail: ylinwang2002@yahoo.com.cn
} 
Methods

Synthesis of cadmium sulfide. The preparation of CdS nanocrystals was carried out in a $250 \mathrm{~mL}$ three-necked flask. $100 \mathrm{~mL}$ of $0.02 \mathrm{M} \mathrm{CdCl}_{2} \cdot 2.5 \mathrm{H}_{2} \mathrm{O}$ was initially added into the flask, then 1.0 $\mathrm{mL}$ thioglycolic acid was introduced under stirring, and the $\mathrm{pH}$ was adjusted to $12.00 \mathrm{using} 1.0$ $\mathrm{M} \mathrm{NaOH}$. After the solution was agitated for $10 \mathrm{~min}, 0.3 \mathrm{~mL}$ of $30 \% \mathrm{H}_{2} \mathrm{O}_{2}$ was added drop wisely into the flask. The reaction mixture was refluxed at $100{ }^{\circ} \mathrm{C}$ under open-air condition, an aliquot of sample was taken out at different times during the reflux.

Characterization. The transmission electron microscopy (TEM) and high-resolution transmission electron microscope (HRTEM) images were performed with a JEM-3010 electron microscope. (JEOL, Japan). X-ray powder diffraction (XRD) was performed using a Rigaku $\mathrm{D} / \mathrm{max} 2500 \mathrm{~V}$ diffractometer (Rigaku, Japan) equipped with a $\mathrm{Cu}$ target. Fourier transform infrared (FTIR) spectra were recorded on a Nexus-470 spectrometer (Nicolet, USA). Photoluminescence measurement was conducted on a RF-5301PC spectrophotometer (Shimadzu, Japan). Absorption spectra were recorded on a UV-2102 spectrometer (Unico (Shanghai), China).

Measurement of quantum yields. The quantum yield (QY) of CdS NCs was measured according to the method described in Ref. [12], briefly, rhodamine6G was chosen as a reference standard $(\mathrm{QY}=95 \%$, in ethanol), the absorbance for the standard and the CdS NCs samples at the excitation wavelength and the fluorescence spectra of the same solutions were measured, respectively. The expression to calculate quantum yield is provided as following:

$$
Y_{\mathrm{u}}=Y_{\mathrm{s}} \times \frac{F_{\mathrm{u}} \times A_{\mathrm{s}}}{F_{\mathrm{s}} \times A_{\mathrm{u}}}
$$

where the subscripts $s$ and $u$ denote standard (such as rhodamine 6G) and test samples, respectively, $Y$ is quantum yield, $F$ is the integrated emission peak area, and $A$ is the absorbance at excitation wavelength. The excitation wavelength for measurements of the QY was set at 365 nm.

\section{RESULTS AND DISCUSSION}

\section{$X$-ray diffraction analysis}

The powder XRD pattern of CdS NCs refluxed for 2 hours is presented in Figure 1. The results of XRD pattern reveal that the product is well crystallized since the diffraction peaks were strong and sharp. Moreover, three typical distinct features of the zinc-blende structure can be distinguished: the one at $2 \theta=26.43^{\circ}$ is due to the (111) reflection, and the other two appearing at $2 \theta=43.84^{\circ}$ and $51.96^{\circ}$ result from the (220) and (311) reflections, respectively. The positions of diffraction peaks match well with the theoretical values of the cubic structure of CdS (JCPDS No.65-2887) with the lattice parameter of $\mathrm{a}=\mathrm{b}=\mathrm{c}=5.832 \AA$. The average size of the CdS NCs was estimated by the Debye-Scherrer formula, and the diameter is about $3.6 \mathrm{~nm}$, which demonstrates that the product is a real nanocrystal.

\section{Fourier transform infrared spectroscopy analysis}

To further confirm the formation of CdS NCs and investigate the interaction between TGA and CdS NCs, the FTIR spectrometry was measured. As shown in Figure 2, a broad absorption band 
around $3400 \mathrm{~cm}^{-1}$ is assigned to $\mathrm{O}-\mathrm{H}$ vibration of the absorbed $\mathrm{H}_{2} \mathrm{O}$. An absorption band due to $\mathrm{S}-\mathrm{H}$ vibration at $2560 \mathrm{~cm}^{-1}$ is absent. The characteristic absorption band of $\mathrm{C}=\mathrm{O}$ vibration shifts from $1700 \mathrm{~cm}^{-1}$ to $1556 \mathrm{~cm}^{-1}$. Therefore, these results strongly suggest that the thiol groups of TGA coordinate with $\mathrm{Cd}^{2+}$ ions on the NCs surface, and the hydrophilic hydroxyl groups face outward, making NCs water-soluble.

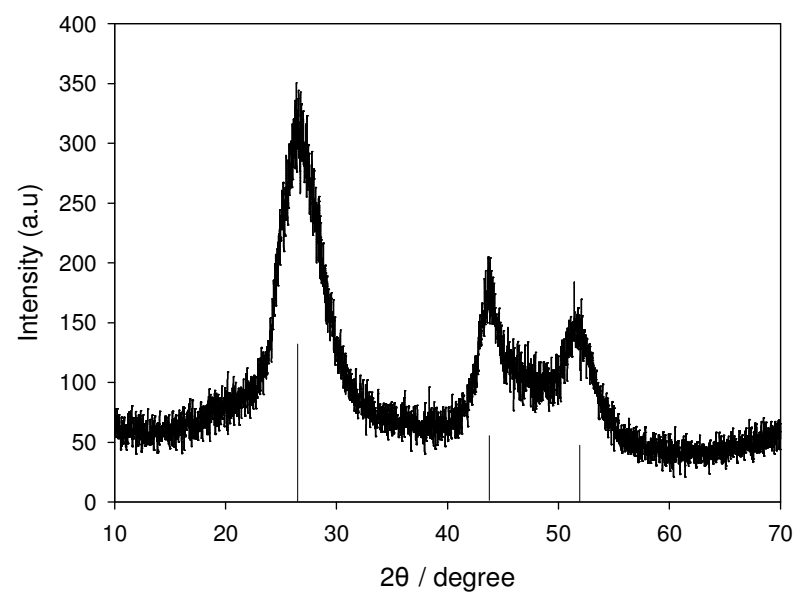

Figure 1. XRD pattern of CdS NCs.

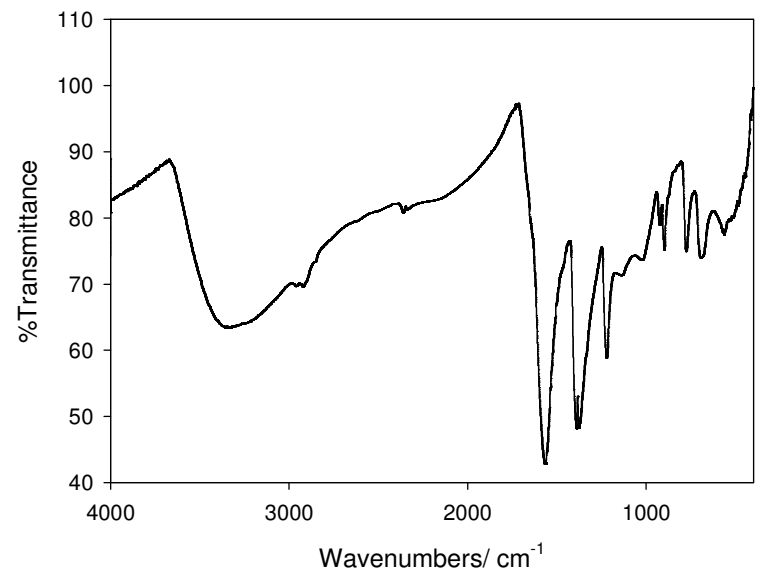

Figure 2. FTIR spectra of TGA-capped CdS NCs.

Transmission electron microscopy analysis

Figure 3A shows the TEM image of CdS NCs synthesized for $120 \mathrm{~min}$. The TEM image confirmed that the size distribution of the as-synthesized CdS NCs were nearly monodisperse with the average size of about $5.0 \mathrm{~nm}$, which is in accordance with the diameter calculated by 
Debye-Scherrer formula. The existence of lattice planes on the HRTEM (Figure 3B) disclosed the good crystallinity of the CdS NCs.
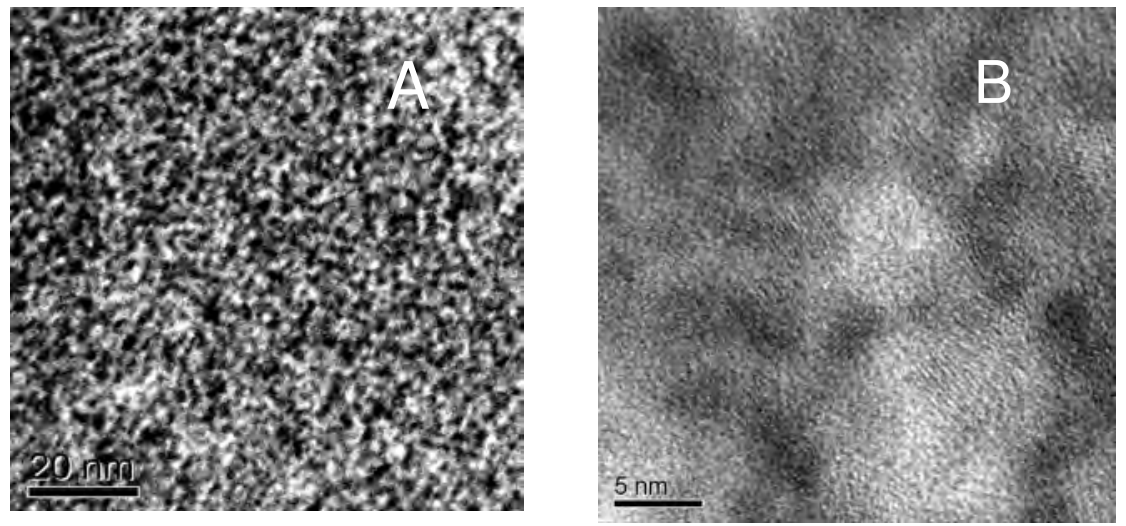

Figure 3. The TEM (a) and HRTEM (b) images of CdS NCs.

\section{Optical properties analysis}

It is well known that bulk CdS started to absorb the light of wavelength of about $515 \mathrm{~nm}$. With the decrease of the size of CdS particle, the absorption threshold shifts to shorter wavelengths as a result of quantum confinement effect [13]. Figure 4A presents the UV-Vis absorption spectra of CdS NCs taken at different refluxing times (15 min and $120 \mathrm{~min}$, respectively). It can be seen that the wavelength of the absorption onset of the two samples is at around $370 \mathrm{~nm}$ and $410 \mathrm{~nm}$, respectively. As expected, the absorbance edge of CdS NCs is blue shifted relative to that of bulk CdS at $515 \mathrm{~nm}$ [14], and the size of CdS NCs increases with the increase of refluxing time.

The investigation demonstrated the photoluminescence spectra of semiconductor nanocrystals originated from electrons in the conduction band, excitonic states and trap states [15-17]. In the case of CdS NCs, it was reported $[18,19]$ that the high energy emission band $(350-500 \mathrm{~nm})$ was due to the radiative recombination of free charge carriers or excitonic fluorescence while low energy PL peak (500-700 nm) was attributed to the recombination of charge carriers in deep traps of surface defect states. As seen in Figure 4B, in our experiment, the emission peaks of the two CdS NCs samples are located at $518 \mathrm{~nm}$ and $610 \mathrm{~nm}$, respectively, which present some blue-shift compared to bulk CdS at $650 \mathrm{~nm}$ [20]. It is obvious that the recombination of charge carriers in deep traps of surface defect states is responsible for the observed PL spectra. With the increase of refluxing time, the peak position is red-shifted and the particle size is increased, which is in agreement with the red-shift of absorption band edge (Figure 4A). The room temperature photoluminescence quantum yields for samples (a) and (b) are $12.6 \%$ and $1.1 \%$, respectively, almost comparable to the CdS NCs prepared by an organometallic route [21]. Moreover, the $\mathrm{CdS}$ NCs are very stable in the dark at low temperature. After the sample was kept in a refrigerator for 30 days, its photoluminescence spectra almost had no change, and no obvious precipitates were observed. 

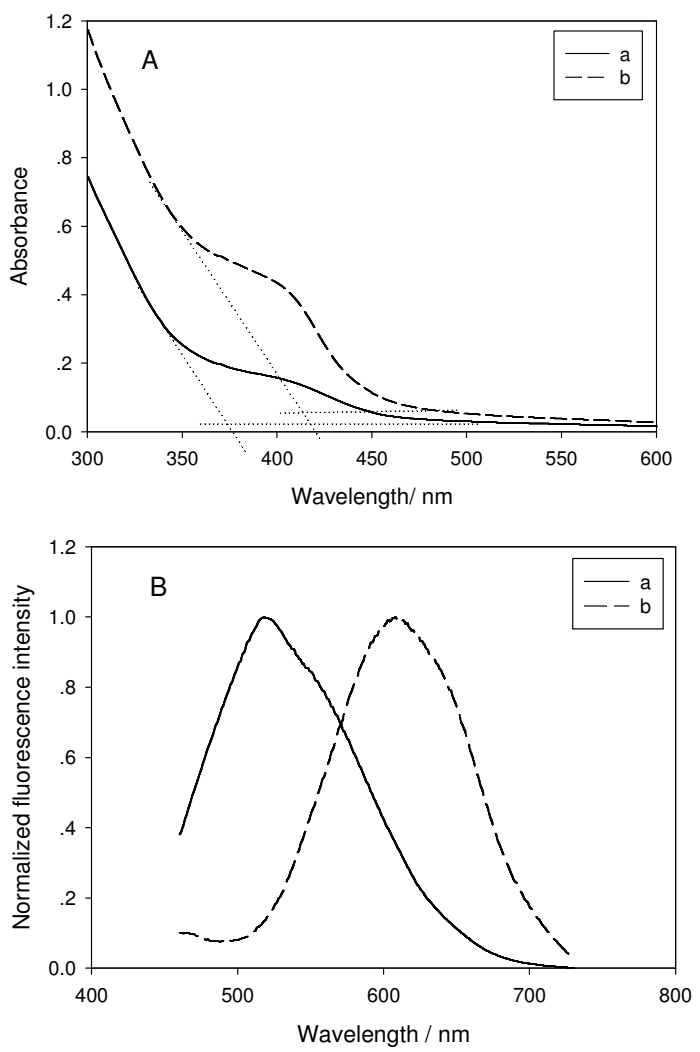

Figure 4. UV-Vis absorption (A) and PL (B) spectra of CdS NCs taken at different refluxing time (a $15 \mathrm{~min}$, b $120 \mathrm{~min}$ ).

\section{Mechanism of the CdS NCs formation}

Based on the above results, the following mechanism is proposed to explain the formation of CdS NCs. First, in strong alkaline condition, $\mathrm{Cd}^{2+}$ ions were coordinated with TGA to form cadmium thiol complexes. Because the TGA was excessive, after the addition of $\mathrm{H}_{2} \mathrm{O}_{2}$ and heating, some TGA molecules reacted with $\mathrm{H}_{2} \mathrm{O}_{2}$ to release $\mathrm{S}^{2-}$ ions. Subsequently, the produced $\mathrm{S}^{2-}$ ions reacted with $\mathrm{Cd}^{2+}$ ions to generate $\mathrm{CdS}$ NCs. The formation processes can be described as follows:

$$
\begin{aligned}
& \mathrm{Cd}^{2+}+\mathrm{TGA} \longrightarrow \mathrm{TGA}-\mathrm{Cd}^{2+} \\
& \stackrel{\mathrm{SH}}{\left.\right|_{\mathrm{CH}}} \stackrel{\|}{\mathrm{C}}-\mathrm{OH} \stackrel{\mathrm{OH}^{-}}{\longrightarrow} \stackrel{\mathrm{SH}}{\mathrm{CH}}=\stackrel{\mathrm{CH}}{\mathrm{C}}-\mathrm{O}^{-} \stackrel{\mathrm{OH}^{-}}{\longrightarrow} \mathrm{S}^{2-}+\mathrm{CH}_{2}=\stackrel{\mathrm{OH}}{\mathrm{C}}-\mathrm{O}^{-} \\
& \mathrm{CH}_{2}=\stackrel{\mathrm{OH}}{\mathrm{C}}-\mathrm{O}^{-} \stackrel{\mathrm{H}_{2} \mathrm{O}_{2}}{\longrightarrow} \mathrm{CO}_{2}+\mathrm{H}_{2} \mathrm{O} \\
& \mathrm{S}^{2-}+\mathrm{TGA}^{-} \mathrm{Cd}^{2+} \longrightarrow \mathrm{TGA}-\mathrm{CdS}
\end{aligned}
$$

Bull. Chem. Soc. Ethiop. 2011, 25(3) 


\section{CONCLUSIONS}

In summary, a novel method has been developed first time to synthesize CdS NCs using TGA as a sulfur source and stabilizer with the presence of hydrogen peroxide in aqueous solution. The obtained CdS NCs have good crystallizability, favorable monodispersity, moderate QY and good stability.

\section{ACKNOWLEDGEMENTS}

This research was supported by the National Natural Science Foundation (61066006) and the Scientific Research Foundation of Guangxi University (XBZ110359).

\section{REFERENCES}

1. Murray, C.B.; Norris, D.J.; Bawendi, M.G. J. Am. Chem. Soc. 1993, 115, 8706.

2. Peng, Z.A.; Peng, X.G. J. Am. Chem. Soc. 2001, 123, 183.

3. Takaaki, T.; Kensuke, A.; Hidemi, N. Langmuir 2004, 20, 11169.

4. Vossmeyer, T.; Katsikas, L.; Gienig, M.; Popovic, I.G.; Diesner, K.; Weller, H. J. Phys. Chem. 1994, 98, 7665.

5. Zhu, J.J.; Zhou, M.G.; Xu, J.Z.; Liao, X.H. Mater. Lett. 2001, 47, 25.

6. Curri, M.L.; Leo, G.; Alvisi, M.; Agostiano, A.; Della Monica, M.; Vasanelli, L. J. Colloid Interf. Sci. 2001, 243, 165.

6. Yao, S.W.; Han, Y.X.; Liu, W.X.; Zhang, W.G.; Wang, H.Z. Mater. Chem. Phys. 2007, 101, 247.

7. Wang, H.M.; Chen, Z.; Fang, P.F.; Wang, S.J. Mater. Chem. Phys. 2007, 106, 443.

8. Wang, Y.L.; Lu, J.P.; Huang, X.F.; Long, J.X.; Tong, Z.F. Mater. Lett. 2008, 62, 3413.

9. Zou, L.; Fang, Z.; Gu, Z.Y.; Zhong, X.H. J. Lumin. 2009, 129, 536.

10. Cheng, X.J.; Zhao, Q.; Yang, Y.K.; Tjong, S.C.; Robert, K.Y. J. Colloid Interf. Sci. 2008, 326, 121.

11. Liu, J.W.; Zhang, Y.; Ge, C.W.; Jin, Y.L.; Hu, S.L.; Gu, N. Chin. Chem. Lett. 2009, 20, 977.

12. Zhang, M.F.; Drechsler, M.; Axelr, H.E. Chem. Mater. 2004, 16, 537.

13. Hwang, S.H.; Moorefield, C.N.; Wang, P.S.; Jeong, K.U.; Cheng, S.Z.D.; Kotta, K.K.; Newkome, G.R. Chem. Commun. 2006, 3495.

14. Skinner, D.E.; Colombo, D.P.; Cavaleri, J.J.; Bowman, R.M. J. Phys. Chem. 1995, 99, 7853.

15. Sengupta, A.; Jiang, B.; Mandal, K.C.; Zhang, J.Z. J. Phys. Chem. B 1999, 103, 3128.

16. Brelle, M.C.; Zhang, J.Z.; Nguyen, L.; Mehra, R.K. J. Phys. Chem. A 1999, 103, 10194.

17. Unni, C.; Philip, D.; Gopchandran, K. Spectrochim. Acta. A 2008, 71, 1402.

18. Chestnoy, N.; Harris, T.D.; Hull, R.; Brus, L.E. J. Phys. Chem. 1986, 90, 3393.

19. Zhang, B.C.; Shen, Y.H.; Xie, A.J.; Yang, L.B.; Wang, X.F. Mater. Chem. Phys. 2009, 116, 392.

20. Cao, Y.C.; Wang, J.H. J. Am. Chem. Soc. 2004, 126, 14336. 